\title{
Robotic stereotactic body radiation therapy for elderly medically inoperable early-stage non-small cell lung cancer
}

This article was published in the following Dove Press journal:

Lung Cancer:Targets and Therapy

6 August 2013

Number of times this article has been viewed

\author{
Sana D Karam' \\ Zachary D Horne' \\ Robert L Hong ${ }^{1,2}$ \\ Nimrah Baig' \\ Gregory J Gagnon ${ }^{4}$ \\ Don McRae ${ }^{2}$ \\ David Duhamel ${ }^{3}$ \\ Nadim M Nasr ${ }^{1,2}$ \\ 'Department of Radiation Oncology, \\ Georgetown University Hospital, \\ Washington, DC, USA; ${ }^{2}$ Department \\ of Radiation Oncology, Virginia \\ Hospital Center, Arlington, VA, \\ USA; ${ }^{3}$ Department of Pulmonary/ \\ Critical Care Medicine, Virginia \\ Hospital Center, Arlington, VA, USA; \\ ${ }^{4}$ Department of Radiation Oncology, \\ Frederick Memorial Hospital, \\ Frederick, MD, USA
}

Introduction: Stereotactic body radiation therapy (SBRT) is being increasingly applied in the treatment of non-small cell lung cancer (NSCLC) because of its high local efficacy. This study aims to examine survival outcomes in elderly patients with inoperable stage I NSCLC treated with SBRT.

Methods: A total of 31 patients with single lesions treated with fractionated SBRT from 2008 to 2011 were retrospectively analyzed. A median prescribed dose of 48 Gy was delivered to the prescription isodose line, over a median of four treatments. The median biologically effective dose (BED) was 105.6 (range 37.50-180), and the median age was 73 (65-90 years). No patient received concurrent chemotherapy.

Results: With a median follow up of 13 months (range, 4-40 months), the actuarial median overall survival (OS) and progression-free survival (PFS) were 32 months, and 19 months, respectively. The actuarial median local control (LC) time was not reached. The survival outcomes at median follow up of 13 months were $80 \%, 68 \%$, and $70 \%$ for LC, PFS, and OS, respectively. Univariate analysis revealed a BED of $>100$ Gy was associated with improved LC rates $(P=0.02)$, while squamous cell histology predicted for worse LC outcome at median follow up time of 13 months $(P=0.04)$. Increased tumor volume was a worse prognostic indicator of both LC and OS outcomes $(P<0.05)$. Finally, female gender was a better prognostic factor for OS than male gender $(P=0.006)$. There were no prognostic indicators of PFS that reached statistical significance. No acute or subacute high-grade toxicities were documented.

Conclusion: SBRT is a safe, feasible, and effective treatment option for elderly patients with inoperable early stage NSCLC. BED, histology, and tumor size are predictors of local control, while tumor size and gender predict OS.

Keywords: SABR, CyberKnife, BED, gender, histology

\section{Introduction}

The standard treatment for stage I non-small cell lung cancer (NSCLC) is surgical resection. ${ }^{1,2}$ Definitive anatomic resection carries a favorable local control (LC) rate and overall survival (OS). ${ }^{1}$ Some patients, however, are medically inoperable because of an unacceptable risk of operative complications and mortality. ${ }^{3,4}$ Alternative treatment options have traditionally included limited surgical resection ${ }^{5}$ or conventional radiotherapy. ${ }^{6}$ Both approaches, however, yield poorer outcomes. ${ }^{5,6}$ Five-year survival rates of $45 \%-59 \%$ and $0 \%-42 \%$ are generally expected from limited surgical resection and conventional radiotherapy treatment, respectively, for inoperable stage I NSCLC. ${ }^{7,8}$

Stereotactic body radiation therapy (SBRT) was introduced for the treatment of inoperable early stage NSCLC over a decade ago. ${ }^{9}$ Using image guidance, SBRT
Correspondence: SD Karam

Department of Radiation Oncology,

Georgetown University Hospital,

3800 Reservoir Rd, NW,

Washington, DC 20007, USA

Tel + I 2024443320

Fax + I 2024449323

Emailsanadkaram@yahoo.com 
allows for the reduction of dose to critical structures thus enabling the delivery of much higher doses to the target. ${ }^{10}$ LC outcomes from early SBRT studies have been confirmed in two recent multicenter Phase II SBRT trials. ${ }^{11,12}$ Timmerman et al reported a primary tumor control rate of $97 \%$ and LC (in the involved lobe) of $91 \%$ at 3 years ${ }^{11}$ following SBRT, whereas Baumann et al reported a 3-year LC rate of $92 \% .{ }^{12}$ OS rates with such techniques have been poor, but this has been attributed to medical comorbidities rather than disease progression or treatment-related toxicity. ${ }^{9-11}$

The CyberKnife system (Accuray Inc, Sunnyvale, CA, USA) integrates a robotically positioned linear accelerator (LINAC) with image-guided stereotactic localization. Its ability to dynamically track targets that move with breathing, with the Synchrony tumor tracking component, is a key feature of this system. ${ }^{13}$ Retrospective studies using this system have shown favorable outcomes in LC and OS, of $91 \%$ and $75 \%$, respectively. ${ }^{14-30}$ The purpose of the present retrospective analysis was to evaluate the outcomes of patients with surgical high-risk, stage I NSCLC treated at our institution, using robotic hypofractionated SBRT.

\section{Methods}

\section{Eligibility}

After research ethics board approval was obtained, all patients with a diagnosis of clinically staged IA or IB NSCLC (per the American Joint Committee on Cancer, staging manual, 7th edition ${ }^{31}$ ) who were treated between 2008 and 2011 with curative-intent radiotherapy at the Virginia Hospital Center, were identified. Patients who had received hypofractionated radiotherapy were retrospectively reviewed. All such patients were either inoperable, as determined by thoracic surgeons, or had refused surgical intervention. All patients had a histologic diagnosis of NSCLC, and all had available a history; a physical examination; computed tomography (CT) imaging of the chest, abdomen, and pelvis; whole body positron emission tomography (PET) scan, brain magnetic resonance imaging (MRI) or CT. The exclusion criteria included recurrent lung cancers or lung metastases, metachronous lung cancer, loss to follow up, or postoperative radiation regimens. Patients treated previously with SBRT were not included in the study population. Charts were reviewed to determine patterns of disease failure, toxicity (as defined by the Common Terminology Criteria for Adverse Events, version 3.0), and outcome.

\section{Fiducial placement}

Most patients received fiducial placement (Gold Fiducial Markers, Best Medical International Inc, Springfield, VA, USA) into the adjacent soft tissue for real-time image guidance, either bronchoscopically, or percutaneously: Two to four gold fiducials, $0.8-1 \mathrm{~mm}$ in diameter by $3-7 \mathrm{~mm}$ in length, were usually placed in a noncollinear arrangement for best translational correction of the radiation beam position. To minimize fiducial migration, a 1-2 week period of time was allowed before simulation was commenced, in order to decrease procedural edema and permit fibrosis and fixation of the fiducials.

For lesions that were adjacent to and did not move independently of the spine (five of the 31 patients), spinal tracking (X-Sight ${ }^{\mathrm{TM}}$ Spine Prone Tracking System; Accuray Inc), which uses spine bony landmarks, was utilized instead. Pretreatment digitally reconstructed radiographs (DRRs) were generated from the CT scans. Three-dimensional target displacements and global rotations of the spinal structures were determined by comparing radiographs with the DRRs. Translations and global rotations were aligned during patient setup and corrected during treatment delivery.

\section{Treatment planning}

Fine-cut $(1.25 \mathrm{~mm}) \mathrm{CT}$ was used for targeting and treatment planning. The planning CT scans were done with intravenous (IV) contrast when needed to allow better distinction between tumor and adjacent vessels or atelectasis. The target lesion was outlined by a radiation oncologist and designated as the gross tumor volume (GTV). The target was generally drawn using CT pulmonary windows. However, a soft-tissue window with contrast was sometimes used to avoid inclusion of the adjacent vessels, atelectasis, or mediastinal or chest wall structures, within the GTV. This target included only abnormal CT signal consistent with gross tumor (ie, the GTV and the clinical target volume were identical). PET/CT fusion was done for all patients, and PET data were used in defining the GTV. An additional margin of $5 \mathrm{~mm}$ was added to the GTV to constitute the planning target volume (PTV), but adjustments were made by the treating physician, based on tumor location, proximity of critical structures, and tumor motion during treatment.

\section{Dosimetry}

Three-dimensional noncoplanar beam arrangements were custom designed for each case, to deliver highly conformal 
prescription dose distributions. Generally, more beams were used for larger lesions. As such, prescription lines covering the PTV were typically around the $80 \%$ (but ranging between $60 \%-90 \%$ ) line, rather than the more traditional 95\%-100\%. Higher isodoses (hotspots) were manipulated to occur within the target and not in adjacent normal tissue.

To ensure good coverage, the prescription isodose surface was chosen such that $95 \%$ of the PTV was conformally covered by the prescription isodose, and $99 \%$ of the PTV received a minimum of $90 \%$ of the prescription dose. For target dose heterogeneity, the prescription isodose surface selected had to be $\geq 60 \%$ and $\leq 90 \%$ of the dose at the center of mass of the PTV. Any dose $>105 \%$ of the prescription dose was forced to occur primarily within the PTV itself and not within the normal tissues outside the PTV. Therefore, the cumulative volume of all tissue outside the PTV receiving a dose $>105 \%$ of prescription dose should not have been more than $15 \%$ of the PTV volume. The following critical structures were contoured: the spinal cord, esophagus, brachial plexus, heart, trachea and proximal bronchial tree, whole lung, and skin. The dose tolerance to the critical structures was in accordance with the American Association of Physicists in Medicine Task Group $101 .^{32}$ Treatment conformity was determined in all patients using the New Conformity Index (NCI), which was calculated by the formula: treatment volume $\times$ prescription isodose line/volume of target covered by the prescription isodose line. ${ }^{2}$

\section{Treatment delivery and follow-up studies}

Tumors were actively tracked in real time during treatment using Synchrony ${ }^{\mathrm{TM}}$. For the treatments, the patient wore a tight-fitting elastic vest. Three beacons, emitting visible red light pulsed at $30 \mathrm{~Hz}$, were placed on this vest. The beacon positions were monitored by a camera mounted at the foot end of the patient couch. This camera continuously recorded the position of the markers during the patients' respiration cycle. In parallel, a series of X-ray images of the internal fiducials was taken with the patient breathing freely. These images established the position of the fiducials and thus, the tumor at the time of these images. The time stamps of the beacon data (as captured by the camera) and the location data of the internal fiducials (as determined at the instant of the X-ray images) were synchronized. From a series of these images, a correlation model, between the external and internal positions, was established. Thus, using the instantaneous information from the external beacon, the position of the tumor was calculated. Using this dynamic model, the robot was able to track the tumor motion in real time, while the radiation was delivered. As treatment commenced, X-ray images were taken, either before each therapeutic beam or less frequently, for systematic breathers, updating the model. Clinical examinations and PET/CT imaging were performed at 3-month follow-up intervals from the end of the treatment.

\section{Statistical analysis}

Progression-free survival (PFS) was defined as the time from the first day of SBRT treatment to local, regional, or distant failure or last follow-up visit in living patients without evidence of recurrence or progression. The LC, local failure, regional failure, and distant failures were defined, as previously described elsewhere. ${ }^{33}$ Patients were censored at the time of death. The OS was defined as the time from SBRT treatment until death or the last follow-up visit. Interpretation of the available fluorodeoxyglucose (FDG-PET)/CT and CT scans and correlative clinical examinations were used to assess for response of the treated lesion 3 months after SBRT. Tumor measurements at each follow up were carried out using the Response Evaluation Criteria In Solid Tumors (RECIST), according to which a complete response (CR) is defined as total tumor disappearance and a partial response (PR) is defined as a $30 \%$ or greater decrease in the longest tumor diameter. We defined CR as no evidence of disease in the treatment volume, by both radiographic and direct clinical examination. "No response" was defined as an absence of marked change or increase in the treated lesion. PR was defined as not meeting the criteria for $\mathrm{CR}$ or no response. A univariate analysis was conducted to evaluate the association between each survival outcome, and the following independent variables considered: histology (adenocarcinoma, SCC), gender (male, female), biologically effective dose (BED) $(<100,>100 \mathrm{~Gy})$, T stage $(1,2)$, age (in years), GTV (in cubic centimeters), SBRT dose (in Gy), smoking (in number of pack years), and Karnofsky Performance Status (KPS). All correlations were initially identified by Cox regression analysis and if significant, the logrank test was run for generation of Kaplan-Meier plots, for select factors. Acute toxicities that occurred during treatment or within the first 2 weeks following the end of treatment, including fatigue, chest pain, shortness of breath, cough, hemoptysis, wheezing, and esophagitis, were examined. Radiation pneumonitis was examined as a subacute toxicity in all patients. Analyses were performed in SAS version 9.2 (SAS Institute Inc, Cary, NC, USA). 


\section{Results}

\section{Patient characteristics}

A total of 36 patients with inoperable early stage NSCLC were treated. Five patients were excluded from the analysis: one did not have a biopsy-proven tumor, two patients had no follow-up information, and two patients had two separate lesions. A total of 31 patients with stage I inoperable NSCLC were therefore included in this study. The baseline patient and disease characteristics are listed in Table 1. The group comprised $61 \%$ males and 39\% females, and the median patient age at the time of treatment was 73 (range 65-90). The majority (96\%) of patients were smokers, and the median KPS for all patients was 85 . The histology varied between SCC (35.5\%) and adenocarcinoma (64.5\%). The majority of the tumors were peripheral $(74 \%)$, while $26 \%$ qualified as central tumors. Most patients presented with T1 disease (74\%), with a median tumor volume of $24.05 \mathrm{~cm}^{3}$ (range 3.90-151) and a median maximum diameter of $2.42 \mathrm{~cm}$ (range 1.2-5.0).

\section{Treatment characteristics}

The treatment characteristics are presented in Table 2 . A median prescribed dose of 48 Gy was delivered to the prescription isodose line over a median of four 1- to 2-hour treatments over 5-10 days (median 7 days). The median dose per fraction was $12 \mathrm{~Gy}$ (range 12-20 Gy). The median BED was 105.6 (range 37.50-180). On average (mean and median), $96 \%$ of the PTV was conformally covered by the prescription isodose line (range 94\%-99.85\%). The median NCI was 1.46 (range 1.27-2.69). The median percent of the total lung volume receiving 15 Gy or more was $6.5 \%$. In general, tumors that were centrally located received slightly lower median BED and had better conformity (Table 2). There were no patients that received concurrent chemotherapy.

\section{Clinical outcomes and prognostic factors}

The median follow up was 13 months (4-40), with a median OS of 32 months and a median PFS of 19 months. The median

Table I Patients' characteristics

\begin{tabular}{ll}
\hline Age (median, range) & $(76,65-90)$ \\
KPS (median, range) & $(70,60-100)$ \\
Gender (male, female) & $(39 \%, 61 \%)$ \\
Smoker (yes, no) (median, range) & $(96 \%, 4 \%)(40,20-100)$ \\
Histology (adenocarcinoma, squamous) & $(64.5 \%, 35.5 \%)$ \\
Clinical stage (TINO, T2N0) & $(74 \%, 26 \%)$ \\
Maximum diameter, cm (mean, median, range) & $(2.42,2.50,1.2-5.0)$ \\
Volume, cc (mean, median, range) & $(36.15,24.05,3.90-15 \mathrm{I})$ \\
\hline
\end{tabular}

Abbreviations: KPS, Karnofsky Performance Status.
Table 2 Treatment characteristics for all patients and subdivided by tumor location (peripheral, central)

\begin{tabular}{ll}
\hline & Mean, median (range) \\
\hline Prescribed dose on all patients, Gy & $49.03,48.00(25-60)$ \\
Peripheral lesions & $47.97,48.00(30-60)$ \\
Central lesions & $44.67,48.00(25-50)$ \\
Prescription IDL on all patients, \% & $82.55,82.00(74-93)$ \\
Peripheral lesions & $83.59,83.00(74-92)$ \\
Central lesions & $82.00,81.00(74-93)$ \\
Number of fractions on all patients & $4.09,4.00(3-5)$ \\
Peripheral lesions & $4.26,4.00(3-5)$ \\
Central lesions & $4.56,5.00(4-5)$ \\
Dose per fraction on all patients, Gy & $12.8,12.00(5-20)$ \\
Peripheral lesions & $11.77,12.00(6-20)$ \\
Central lesions & $10.00,10.00(5-10)$ \\
BED on all patients & $115.27,105.60(37.50-180)$ \\
Peripheral lesions & $106.15,105.60(48-180)$ \\
Central lesions & $91.04,100.00(37.50-105.60)$ \\
Coverage on all patients & $96.42,96.30(94.30-99.85)$ \\
Peripheral lesions & $96.34,96.00(94.30-99.30)$ \\
Central lesions & $96.15,96.40(94.30-97.10)$ \\
NCl on all patients & $1.56,1.45(1.27-2.69)$ \\
Peripheral lesions & $1.57,1.53(1.27-2.69)$ \\
Central lesions & $1.43,1.43(1.30-1.58)$ \\
\hline
\end{tabular}

Notes: The $\mathrm{NCl}$ is calculated by the formula: treatment volume $\times$ prescription isodose line/volume of target covered by prescription isodose line. ${ }^{2}$

Abbreviations: BED, biologically effective dose; IDL, isodose line; $\mathrm{NCl}$, New Conformity Index.

LC was not reached. The 1-year OS, PFS, LC, and regional control rates were $70 \%, 68 \%, 80 \%$, and $80 \%$, respectively (Table 3 and Figure 1). Seven patients failed locally, and five of them were salvaged with SBRT reirradiation and to date, are alive, while two of the local failures died of the disease. Univariate analysis revealed that a BED of $>100$ Gy was associated with improved LC rates $(P=0.02)$. Histology was also a predictor of LC, with improved LC outcomes in tumors of adenocarcinomatous histology $(P=0.04)$ (Figure 2). Increased tumor volume was also a worse prognostic indicator for both $\mathrm{LC}$ and $\mathrm{OS}(P<0.05)$. Female gender was a better prognostic factor for OS than male gender $(P=0.006)$

Table 3 Clinical outcomes with actuarial rates at median follow up of 13 months

\begin{tabular}{ll}
\hline Median follow up (range in months) & $13(4-40)$ \\
Local control (\%) & $24 / 3 \mid(80 \%)$ \\
Regional control (\%) & $26 / 3 \mid(88 \%)$ \\
Distant control (\%) & $25 / 3 \mid(84 \%)$ \\
Any progression (\%) & $10 / 31(68 \%)$ \\
Overall survival (\%) & $21 / 3 \mid(70 \%)$ \\
\hline
\end{tabular}


A
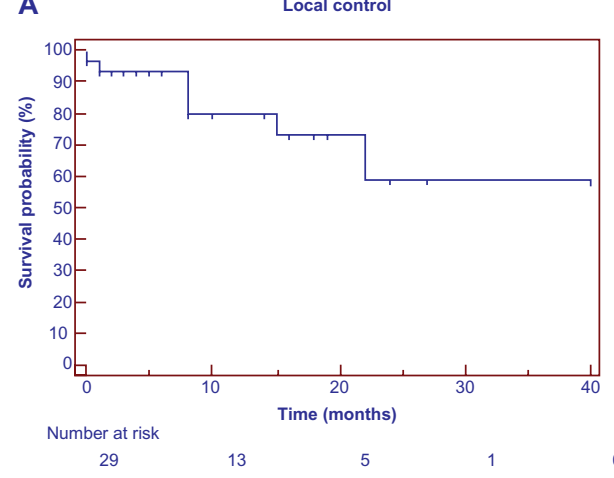

C

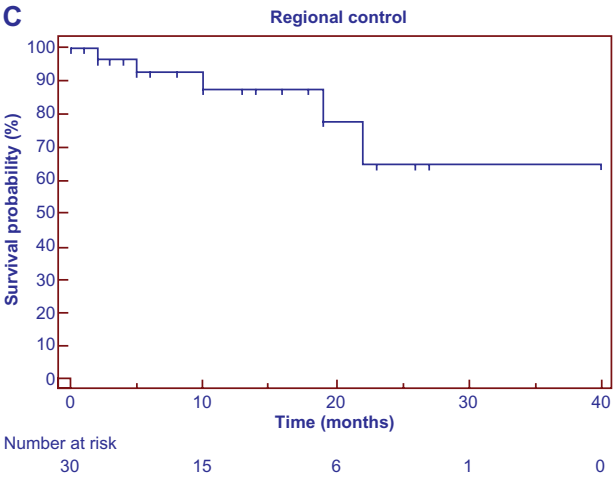

B

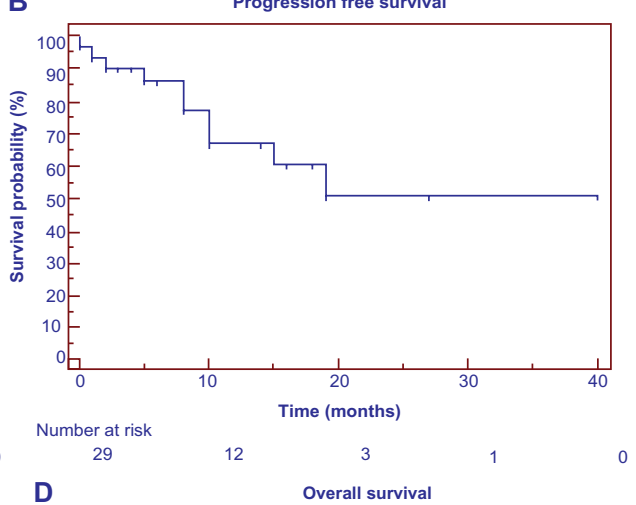

D

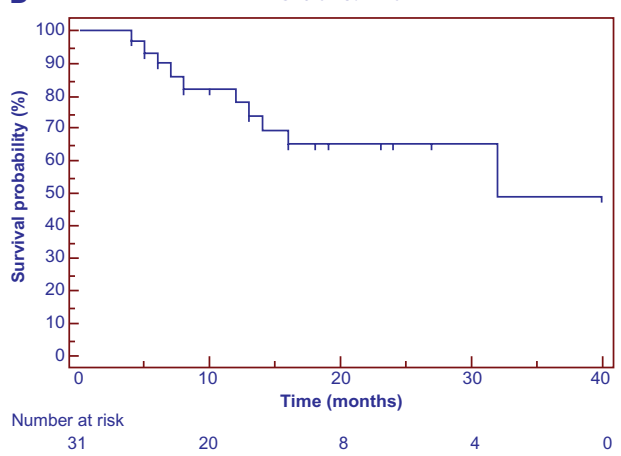

Figure I Survival outcomes of elderly inoperable patients with early stage lung cancer $(n=35) ;(\mathbf{A})$ local control; (B) progression-free survival; (C) regional control; (D) overall survival.

(Figure 3). There were no prognostic indicators of PFS that reached statistical significance.

\section{Toxicity}

Acute toxicities experienced by this patient population included fatigue that was experienced by four patients, and cough and chest pain that were reported by two patients. These symptoms self-resolved and did not require any medical intervention. Three patients experienced shortness of breath in the acute setting that was alleviated with bronchodilators. None of the patients experienced pneumonitis, esophagitis, or hemoptysis.

\section{Discussion}

For early stage patients who are medically inoperable, SBRT is becoming the standard of care. ${ }^{9}$ The present study described

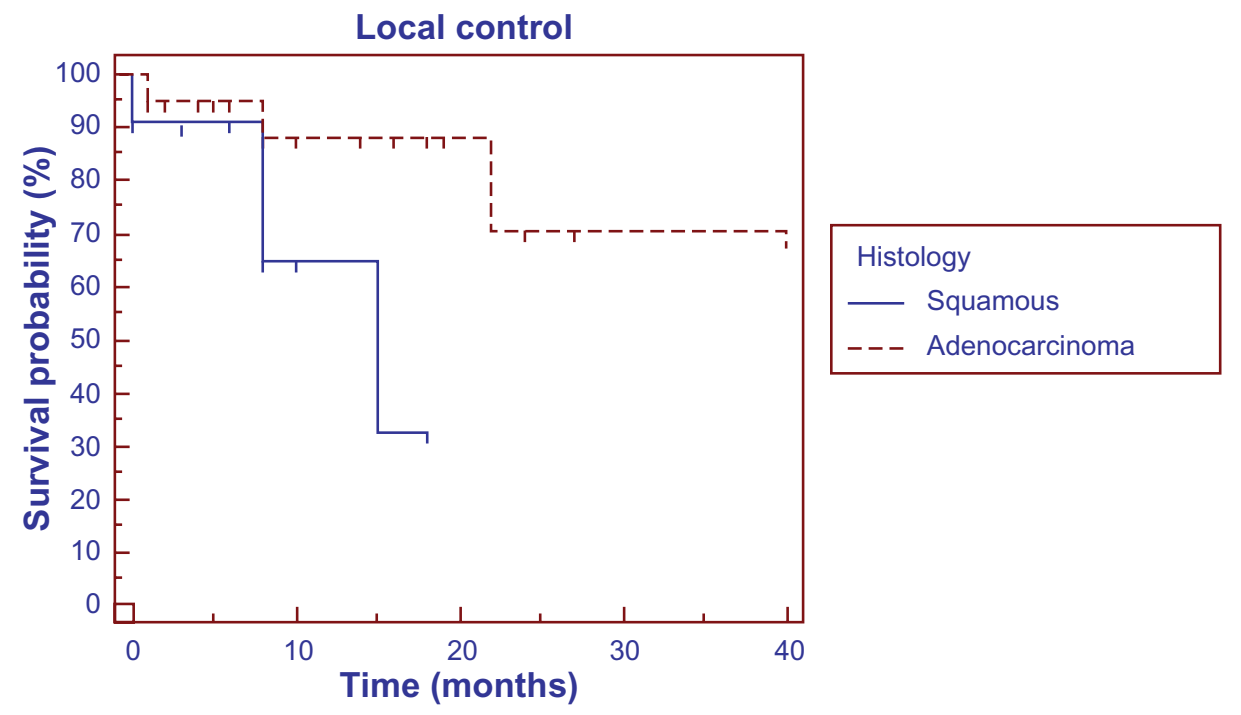

Figure 2 Local control and histology. 


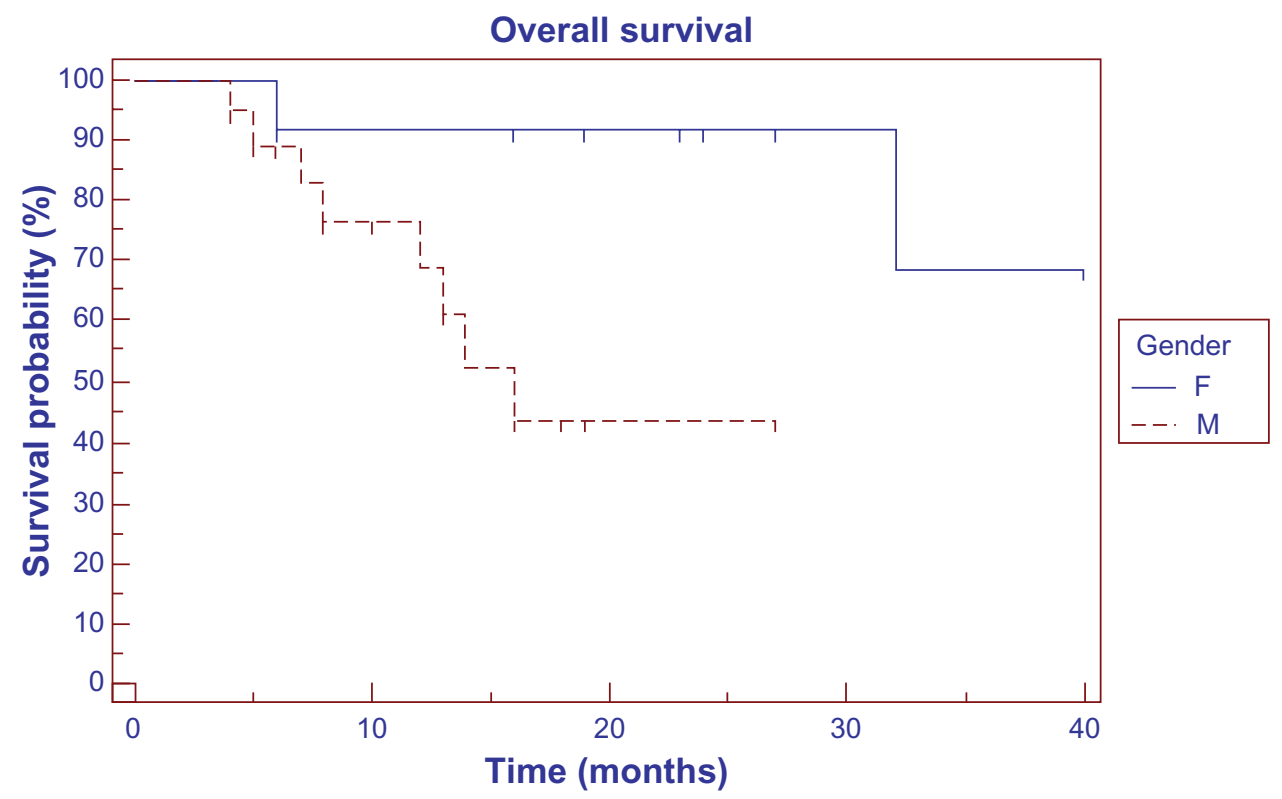

Figure 3 Overall survival and gender.

our recent experience with the definitive treatment of earlystage NSCLC inoperable elderly patients, using stereotactic radiosurgery with the CyberKnife system. The unique ability to dynamically track targets that move with breathing is a key feature that differentiates the CyberKnife from other commercially available image-guided platforms, which typically use either respiratory manipulation or respiratory gating in order to manage breathing-induced motion. The literature on SBRT use for early stage lung cancer reports 3 -year LC and OS rates of $80 \%-98 \%$ and $43 \%-72 \%$, respectively ${ }^{9,11,34,35}$ Timmerman et al reported a 3-year LC rate of $91 \%-92 \%$ in two recent multicenter Phase II SBRT trials. ${ }^{11,36}$ Similar LC outcomes have been reported with the CyberKnife system. ${ }^{140}$ Our survival outcomes demonstrate equivalent $\mathrm{OS}$ rates but lower LC rates $(80 \%)$ than that reported in the literature using this system. This could be due to the inherent heterogeneity between patient populations studied but could also be related to the fact that when SBRT was first utilized at our center, the dosing and the BED in particular were suboptimal.

Similar to previous reports, our results show that a BED of $>100$ Gy is a strong predictor of improved LC. With SBRT, the cumulative BED is significantly higher than that used with conventional radiation treatment. This high-precision SBRT delivery system allows for a sharp dose gradient at the edge of the target volume that enables the delivery of an ablative dose to the target, while minimizing treatment toxicity to a tolerable level. A BED above 100 Gy has been used as a cutoff for adequate dose; below this threshold, the local recurrence risk is higher. ${ }^{37-39} \mathrm{~A}$ recent meta analysis showed that a medium to medium-high BED, defined as BED range 83.2-146 Gy, correlated with improved survival in early stage NSCLC patients. ${ }^{40}$ Our results also show a negative correlation between tumor volume, LC, and OS. This is consistent with the published literature demonstrating that the rates of local recurrence rates increase as tumor size increases. ${ }^{37,39,41,42}$

Our results show a correlation between a tumor histology of adenocarcinoma origin and improved LC rate. The association between tumor histology and radiation response has long been discussed, and it is generally believed that SCC is generally more responsive to radiation treatment. ${ }^{43} \mathrm{~A}$ large multicenter retrospective analysis has shown patients with adenocarcinomatous histology to have significantly better overall prognosis to radiation treatment than those with squamous histology. ${ }^{44}$ However, a recent prospective study examining the difference in SBRT response between the two histologies showed that although tumor shrinkage was faster for SCC than adenocarcinomas at 2 and 4 months, there was no difference in the mean tumor size at 6 months or in the 3 -year LC. ${ }^{45}$ Our results are likely biased by the small sample size and the low event rate; caution is therefore exercised in extrapolating any definitive conclusions from the data.

The strong correlation between female gender and improved OS reaffirms previously published results. In an analysis aimed at investigating the prognostic factors associated with improved survival outcomes in early stage NSCLC treated with SBRT, increased tumor diameter and 
male gender were the only significant variables that negatively influenced OS on multivariate analysis. ${ }^{42}$ Furthermore, a recursive partitioning analysis model showed gender to have a significant effect on OS outcome. ${ }^{42}$ Similarly, a retrospective review of 831 nonmetastatic NSCLC patients treated with external beam radiation therapy (EBRT) at the MD Anderson Cancer Center showed gender to be an independent prognostic factor associated with survival outcomes, ${ }^{46}$ among cases of medically inoperable Stage I NSCLC, the female patients had a better 5-year OS than did male patients (30.0\% versus $13.1 \%$, respectively). ${ }^{46}$ Although our data agree with these published results, our sample size is comparatively small and limits the ability to draw any firm conclusion on the clinical significance of gender and survival outcomes in this patient population.

\section{Conclusion}

This study has demonstrated that the use of SBRT in inoperable, elderly patients with early stage NSCLC is well tolerated and provides acceptable LC outcomes. Our retrospective review is limited by potential selection bias, small sample size, and heterogeneous patient population and treatment parameters, and many issues remain to be elucidated. Our study lacked data on ethnic and racial background, and the impact of ethnicity and racial disparities on survival outcomes deserves investigation, especially as SBRT becomes a widely accepted application in early-stage NSCLC. Randomized, controlled trials are warranted in order to draw firm conclusions about the clinical benefit of SBRT in the treatment of this disease.

\section{Acknowledgment}

We thank Dr James W Snider with assistance in data collection.

\section{Disclosure}

Dr Gagnon is on the clinical advisory board for US Radiosurgery. Actual or potential conflicts of interest do not exist for any other authors.

\section{References}

1. Ginsberg RJ, Rubinstein LV. Randomized trial of lobectomy versus limited resection for T1 N0 non-small cell lung cancer. Lung Cancer Study Group. Ann Thorac Surg. 1995;60(3):615-622.

2. Shirvani SM, Jiang J, Chang JY, et al. Comparative effectiveness of 5 treatment strategies for early-stage non-small cell lung cancer in the elderly. Int J Radiat Oncol Biol Phys. 2012;84(5):1060-1070.

3. Handy JR Jr, Asaph JW, Skokan L, et al. What happens to patients undergoing lung cancer surgery? Outcomes and quality of life before and after surgery. Chest. 2002;122(1):21-30.

4. Allen MS, Darling GE, Pechet TT, et al. Morbidity and mortality of major pulmonary resections in patients with early-stage lung cancer: initial results of the randomized, prospective ACOSOG Z0030 trial. Ann Thorac Surg. 2006;81(3):1013-1019.
5. Narsule CK, Ebright MI, Fernando HC. Sublobar versus lobar resection: current status. Cancer J. 2011;17(1):23-27.

6. Qiao X, Tullgren O, Lax I, Sirzén F, Lewensohn R. The role of radiotherapy in the treatment of stage I non-small cell lung cancer. Lung Cancer. 2003;41(1):1-11.

7. Rowell NP, Williams CJ. Radical radiotherapy for stage I/II non-small cell lung cancer in patients not sufficiently fit for or declining surgery (medically inoperable): a systematic review. Thorax. 2001;56(8):628-638.

8. Detterbeck F. A guide to staging in lung cancer, but potentially misleading without attention to the details. Am J Respir Crit Care Med. 2008; 178(2):209-210

9. Palma D, Senan S. Stereotactic radiation therapy: changing treatment paradigms for stage I nonsmall cell lung cancer. Curr Opin Oncol. 2011;23(2):133-139.

10. Grills IS, Hugo G, Kestin LL, et al. Image-guided radiotherapy via daily online cone-beam CT substantially reduces margin requirements for stereotactic lung radiotherapy. Int J Radiat Oncol Biol Phys. 2008;70(4): $1045-1056$.

11. Timmerman R, Paulus R, Galvin J, et al. Stereotactic body radiation therapy for inoperable early stage lung cancer. JAMA. 2010;303(11): 1070-1076.

12. Baumann P, Nyman J, Hoyer M, et al. Outcome in a prospective phase II trial of medically inoperable stage I non-small-cell lung cancer patients treated with stereotactic body radiotherapy. J Clin Oncol. 2009;27(20): 3290-3296.

13. Gibbs IC, Loo BW Jr. CyberKnife stereotactic ablative radiotherapy for lung tumors. Technol Cancer Res Treat. 2010;9(6):589-596.

14. Chen VJ, Oermann E, Vahdat S, et al. CyberKnife with tumor tracking: an effective treatment for high-risk surgical patients with stage I nonsmall cell lung cancer. Front Oncol. 2012;2:9.

15. Brown WT, Wu X, Fayad F, et al. Application of robotic stereotactic radiotherapy to peripheral stage I non-small cell lung cancer with curative intent. Clin Oncol (R Coll Radiol). 2009;21(8):623-631.

16. Le QT, Loo BW, Ho A, et al. Results of a phase I dose-escalation study using single-fraction stereotactic radiotherapy for lung tumors. J Thorac Oncol. 2006;1(8):802-809.

17. Collins BT, Vahdat S, Erickson K, et al. Radical cyberknife radiosurgery with tumor tracking: an effective treatment for inoperable small peripheral stage I non-small cell lung cancer. J Hematol Oncol. 2009;2:1.

18. Pennathur A, Luketich JD, Heron DE, et al. Stereotactic radiosurgery for the treatment of stage I non-small cell lung cancer in high-risk patients. J Thorac Cardiovasc Surg. 2009;137(3):597-604.

19. van der Voort van Zyp NC, Prévost JB, Hoogeman MS, et al. Stereotactic radiotherapy with real-time tumor tracking for non-small cell lung cancer: clinical outcome. Radiother Oncol. 2009;91(3):296-300.

20. Whyte RI, Crownover R, Murphy MJ, et al. Stereotactic radiosurgery for lung tumors: preliminary report of a phase I trial. Ann Thorac Surg. 2003;75(4):1097-1101.

21. Nuyttens JJ, Prevost JB, Praag J, et al. Lung tumor tracking during stereotactic radiotherapy treatment with the CyberKnife: Marker placement and early results. Acta Oncol. 2006;45(7):961-965.

22. Brown WT, Wu X, Fayad F, et al. CyberKnife radiosurgery for stage I lung cancer: results at 36 months. Clin Lung Cancer. 2007;8(8): 488-492.

23. Brown WT, Wu X, Amendola B, et al. Treatment of early non-small cell lung cancer, stage IA, by image-guided robotic stereotactic radioablation - CyberKnife. Cancer J. 2007;13(2):87-94.

24. Brown WT, Wu X, Wen BC, et al. Early results of CyberKnife imageguided robotic stereotactic radiosurgery for treatment of lung tumors Comput Aided Surg. 2007;12(5):253-261.

25. Collins BT, Erickson K, Reichner CA, et al. Radical stereotactic radiosurgery with real-time tumor motion tracking in the treatment of small peripheral lung tumors. Radiat Oncol. 2007;2:39.

26. Muacevic A, Drexler C, Wowra B, et al. Technical description, phantom accuracy, and clinical feasibility for single-session lung radiosurgery using robotic image-guided real-time respiratory tumor tracking. Technol Cancer Res Treat. 2007;6(4):321-328. 
27. Pennathur A, Luketich JD, Burton S, et al. Stereotactic radiosurgery for the treatment of lung neoplasm: initial experience. Ann Thorac Surg. 2007;83(5):1820-1824.

28. Brown WT, Wu X, Fowler JF, et al. Lung metastases treated by CyberKnife image-guided robotic stereotactic radiosurgery at 41 months. South Med J. 2008;101(4):376-382.

29. Ahn SH, Han MS, Yoon JH, et al. Treatment of stage I non-small cell lung cancer with CyberKnife, image-guided robotic stereotactic radiosurgery. Oncol Rep. 2009;21(3):693-696.

30. Vahdat S, Oermann EK, Collins SP, et al. CyberKnife radiosurgery for inoperable stage IA non-small cell lung cancer: $18 \mathrm{~F}$-fluorodeoxyglucose positron emission tomography/computed tomography serial tumor response assessment. J Hematol Oncol. 2010;3:6.

31. Edge SB, Byrd DR, Compton CC, Fritz AG, Greene FL, Trotti A, editors. AJCC Cancer Staging Manual. 7th ed. New York: Springer; 2010.

32. Benedict SH, Yenice KM, Followill D, et al. Stereotactic body radiation therapy: the report of AAPM Task Group 101. Med Phys. 2010;37(8): 4078-4101.

33. Timmerman RD. Surgery versus stereotactic body radiation therapy for early-stage lung cancer: who's down for the count? J Clin Oncol. 2010;28(6):907-909.

34. Koto M, Takai Y, Ogawa Y, et al. A phase II study on stereotactic body radiotherapy for stage I non-small cell lung cancer. Radiother Oncol. 2007;85(3):429-434.

35. Fakiris AJ, McGarry RC, Yiannoutsos CT, et al. Stereotactic body radiation therapy for early-stage non-small-cell lung carcinoma: four-year results of a prospective phase II study. Int J Radiat Oncol Biol Phys. 2009;75(3):677-682.

36. Timmerman R, McGarry R, Yiannoutsos C, et al. Excessive toxicity when treating central tumors in a phase II study of stereotactic body radiation therapy for medically inoperable early-stage lung cancer. J Clin Oncol. 2006;24(30):4833-4839.

37. Onishi H, Shirato H, Nagata Y, et al. Hypofractionated stereotactic radiotherapy (HypoFXSRT) for stage I non-small cell lung cancer: updated results of 257 patients in a Japanese multi-institutional study. J Thorac Oncol. 2007;2(7 Suppl 3):S94-S100.
38. Baumann P, Nyman J, Lax I, et al. Factors important for efficacy of stereotactic body radiotherapy of medically inoperable stage I lung cancer. A retrospective analysis of patients treated in the Nordic countries. Acta Oncol. 2006;45(7):787-795.

39. Onishi H, Araki T, Shirato H, et al. Stereotactic hypofractionated high-dose irradiation for stage I nonsmall cell lung carcinoma: clinical outcomes in 245 subjects in a Japanese multiinstitutional study. Cancer. 2004;101(7):1623-1631.

40. Zhang J, Yang F, Li B, et al. Which is the optimal biologically effective dose of stereotactic body radiotherapy for Stage I non-small-cell lung cancer? A meta-analysis. Int J Radiat Oncol Biol Phys. 2011;81(4): e305-e316.

41. Bradley JD, El Naqa I, Drzymala RE, Trovo M, Jones G, Denning MD. Stereotactic body radiation therapy for early-stage non-small-cell lung cancer: the pattern of failure is distant. Int J Radiat Oncol Biol Phys. 2010;77(4):1146-1150.

42. Matsuo Y, Shibuya K, Nagata Y, et al. Prognostic factors in stereotactic body radiotherapy for non-small-cell lung cancer. Int J Radiat Oncol Biol Phys. 2011;79(4):1104-1111.

43. Tsukahara Y, Sakai Y, Noguchi H, Iwai S, Fukuta T. A study on radiosensitivity and prognostic factors of cervical adenocarcinoma. Nihon Sanka Fujinka Gakkai Zasshi. 1980;32(10):1609-1614.

44. Holgersson G, Bergstöm S, Bergqvist M, et al. Swedish lung cancer radiation study group: predictive value of histology for radiotherapy response in patients with non-small cell lung cancer. Eur J Cancer. 2011;47(16):2415-2421.

45. Miyakawa A, Shibamoto Y, Kosaki K, Hashizume C. Early response and local control of stage I non-small-cell lung cancer after stereotactic radiotherapy: difference by histology. Cancer Sci. 2013;104(1): 130-134.

46. McGovern SL, Liao Z, Bucci MK, et al. Is sex associated with the outcome of patients treated with radiation for nonsmall cell lung cancer? Cancer. 2009;115(14):3233-3242.
Lung Cancer: Targets and Therapy

\section{Publish your work in this journal}

Lung Cancer: Targets and Therapy is an international, peer-reviewed, open access journal focusing on lung cancer research, identification of therapeutic targets and the optimal use of preventative and integrated treatment interventions to achieve improved outcomes, enhanced survival and quality of life for the cancer patient. Specific topics covered in the journalinclude: Epidemion screening; Cellular research and biomarkers; Identification of biotargets and agents with novel Submit your manuscript here: http://www.dovepress.com/lung-cancer-targets--therapy-journa

\section{Dovepress}

mechanisms of action; Optimal clinical use of existing anticancer agents, including combination therapies; Radiation and surgery; Palliative care; Patient adherence, quality of life, satisfaction; Health economic evaluations. The manuscript management system is completely online and includes a very quick and fair peer-review . quotes from published authors. 